\title{
A NON-AUTONOMOUS KIND OF DUFFING EQUATION
}

\author{
JAUME LLIBRE AND ANA RODRIGUES
}

ABSTRACT. We study the periodic solutions of the following kind of non-autonomous Duffing differential equation $\ddot{y}+a y-\varepsilon y^{3}=\varepsilon h(t, y, \dot{y})$, with $a>0, \varepsilon$ a small parameter and $h$ a $\mathcal{C}^{2}$ function in its variables.

\section{IntRoduCtion AND STATEMENT OF THE MAIN RESUlts}

In 1922 Hamel [8] provided the first general results for the existence of periodic solutions of the periodically forced pendulum equation

$$
\ddot{y}+a \sin y=b \sin t \text {. }
$$

This differential equation was the main subject of a monograph published four years earlier by Duffing [5], who had restricted his study to the approximate determination of the periodic solutions for the approximation

$$
\ddot{y}+a y-c y^{3}=b \sin t,
$$

of equation (1), which nowdays is known as the Duffing differential equation. For more information on the history of these differential equations see the paper of Mawhin [13]. The almost 200 references quoted in this last paper are on the periodic solutions of different kind of Duffing equations, and from its publication many new papers working on these type of periodic solutions also have been published, see the papers $[3,4,17]$ and the quoted references therein. See also the non-autonomous differential equation studied in [7].

In a recent paper (see [9]) we studied the following kind of autonomous Duffing differential equations

$$
\ddot{y}+a y-\varepsilon y^{3}=\varepsilon h(y, \dot{y}),
$$

with $a>0, \varepsilon$ a small parameter and $h$ a $\mathcal{C}^{2}$ function in its variables. A question that naturally arises is the study of a non-autonomous version of equation (2).

In this paper we consider a non-autonomous modified Duffing equation of the form

$$
\ddot{y}+a y-\varepsilon y^{3}=\varepsilon h(t, y, \dot{y}),
$$

where again $a>0, \varepsilon$ a small parameter and $h$ a $\mathcal{C}^{2}$ function in its variables.

The averaging method (see for instance [15]) gives a quantitative relation between the solutions of some non-autonomous differential system and the solutions of its autonomous averaged differential system, and in particular allows to study the periodic solutions of the non-autonomous differential system in function of the periodic solutions of the averaged one, see for more details $[1,2,6,10,11,15,16]$ and mainly

2010 Mathematics Subject Classification. 37G15, 37C80, 37C30.

Key words and phrases. Duffing differential equation, periodic solution, averaging theory. 
section 2 of this paper. Our aim is to apply the averaging theory to system (3) for studying its periodic solutions.

We will write the second order differential equation (3) as the system of two differential equations of first order

$$
\begin{aligned}
& \dot{x}=-a y+\varepsilon\left(y^{3}+h(t, x, y)\right), \\
& \dot{y}=x .
\end{aligned}
$$

We define the functions

$$
\begin{aligned}
& f_{1}\left(x_{0}, y_{0}\right)=\frac{3 y_{0}\left(x_{0}^{2}+a y_{0}^{2}\right)}{8 a^{3 / 2}}+\frac{1}{2 \pi} \int_{0}^{2 \pi / \sqrt{a}} \cos (\sqrt{a} t) h\left(t, \tilde{x}_{0}, \tilde{y}_{0}\right) d t \\
& f_{2}\left(x_{0}, y_{0}\right)=-\frac{3 x_{0}\left(x_{0}^{2}+a y_{0}^{2}\right)}{8 a^{5 / 2}}-\frac{1}{2 \pi \sqrt{a}} \int_{0}^{2 \pi / \sqrt{a}} \sin (\sqrt{a} t) h\left(t, \tilde{x}_{0}, \tilde{y}_{0}\right) d t,
\end{aligned}
$$

where

$$
\tilde{x}_{0}=x_{0} \cos \sqrt{a} t-\sqrt{a} y_{0} \sin \sqrt{a} t, \quad \tilde{y}_{0}=\frac{\sqrt{a} y_{0} \cos \sqrt{a} t+x_{0} \sin \sqrt{a} t}{\sqrt{a}} .
$$

We prove the following theorem which is the main result of this paper:

Theorem 1. For $\varepsilon \neq 0$ sufficiently small and for every zero $\left(x_{0}^{*}, y_{0}^{*}\right) \neq(0,0)$ of the system $f_{1}\left(x_{0}, y_{0}\right)=0, f_{2}\left(x_{0}, y_{0}\right)=0$, such that

$$
\operatorname{det}\left(\left.\frac{\partial\left(f_{1}, f_{2}\right)}{\partial\left(x_{0}, y_{0}\right)}\right|_{\left(x_{0}, y_{0}\right)=\left(x_{0}^{*}, y_{0}^{*}\right)}\right) \neq 0,
$$

the non-autonomous Duffing differential system (4) has a periodic solution $(x(t, \varepsilon)$, $y(t, \varepsilon))$ such that when $\varepsilon \rightarrow 0$ it tends to the periodic solution

$$
x(t)=x_{0}^{*} \cos \sqrt{a} t-\sqrt{a} y_{0}^{*} \sin \sqrt{a} t, \quad y(t)=\frac{\sqrt{a} y_{0}^{*} \cos \sqrt{a} t+x_{0}^{*} \sin \sqrt{a} t}{\sqrt{a}}
$$

of system (4) with $\varepsilon=0$. Moreover, if the eigenvalues of the Jacobian matrix

$$
\left(\left.\frac{\partial\left(f_{1}, f_{2}\right)}{\partial\left(x_{0}, y_{0}\right)}\right|_{\left(x_{0}, y_{0}\right)=\left(x_{0}^{*}, y_{0}^{*}\right)}\right)
$$

are hyperbolic, then the corresponding periodic solution $(x(t, \varepsilon), y(t, \varepsilon))$ of system (4) is hyperbolic and its stability or inestability type is given by those eigenvalues.

Theorem 1 is proved in section 3.

Corollary 2. For $\varepsilon \neq 0$ sufficiently small the non-autonomous Duffing equation (4) with $h(t, x, y)=\sin \sqrt{a} t$ has at least 1 periodic solution.

Corollary 3. For $\varepsilon \neq 0$ sufficiently small the non-autonomous Duffing equation (4) with $h(t, x, y)=\left(1+2 x+5 y-5 x^{2}-y^{2}\right) \sin t$ has at least 5 periodic solutions.

Corollary 4. For $\varepsilon \neq 0$ sufficiently small the non-autonomous Duffing equation (4) with $h(t, x, y)=-\sin \left(x^{2}+y^{2}\right) \sin t$ has at least 1 periodic solution.

The proofs of the corollaries are given in section 4 . 


\section{BASIC RESULTS}

In this section we present the basic results from the averaging theory that we shall need for proving the main results of this paper. For a general introduction to the averaging theory and related topics see the books $[1,6,11,15,16]$. But the results that we shall use are presented in what follows.

We consider the problem of the bifurcation of $T$-periodic solutions from the differential system

$$
\dot{\mathbf{x}}(t)=F_{0}(t, \mathbf{x})+\varepsilon F_{1}(t, \mathbf{x})+\varepsilon^{2} F_{2}(t, \mathbf{x}, \varepsilon),
$$

with $\varepsilon=0$ to $\varepsilon \neq 0$ sufficiently small. The functions $F_{0}, F_{1}: \mathbb{R} \times \Omega \rightarrow \mathbb{R}^{n}$ and $F_{2}: \mathbb{R} \times \Omega \times\left(-\varepsilon_{0}, \varepsilon_{0}\right) \rightarrow \mathbb{R}^{n}$ appearing in $(7)$, are $C^{2}$ in their variables and $T$-periodic in the first variable, and $\Omega$ is an open subset of $\mathbb{R}^{n}$. One of the main assumptions is that the unperturbed system

$$
\dot{\mathbf{x}}(t)=F_{0}(t, \mathbf{x})
$$

has an open subset of $\Omega$ fulfilled of periodic solutions. A solution of this problem is given in the following using the averaging theory.

Let $\mathbf{x}(t, \mathbf{z}, \varepsilon)$ be the solution of system $(7)$ such that $\mathbf{x}(0, \mathbf{z}, \varepsilon)=\mathbf{z}$. We write the linearization of the unperturbed system (8) along a periodic solution $\mathbf{x}(t, \mathbf{z}, 0)$ as

$$
\mathbf{y}^{\prime}=D_{\mathbf{x}} F_{0}(t, \mathbf{x}(t, \mathbf{z}, 0)) \mathbf{y},
$$

where $\mathbf{y}$ is an $n \times n$ matrix. In what follows we denote by $M_{\mathbf{z}}(t)$ some fundamental matrix of the linear differential system (9).

We assume that there exists an open set $W$ with $\mathrm{Cl}(W) \subset \Omega$ such that for each $\mathbf{z} \in \mathrm{Cl}(W), \mathbf{x}(t, \mathbf{z}, 0)$ is $T$-periodic. The set $\mathrm{Cl}(W)$ is isochronous for system (7), i.e. it is a set formed only by periodic solutions, all of them having the same period $T$. Then an answer to the problem of the bifurcation of $T$-periodic solutions from the periodic solutions $\mathbf{x}(t, \mathbf{z}, 0)$ contained in $\mathrm{Cl}(W)$ is given in the next result.

Theorem 5. (Perturbations of an isochronous set) We assume that there exists an open and bounded set $W$ with $\mathrm{Cl}(W) \subset \Omega$ such that for each $\mathbf{z} \in \mathrm{Cl}(W)$, the solution $\mathbf{x}(t, \mathbf{z}, 0)$ is $T$-periodic, then we consider the function $\mathcal{F}: \mathrm{Cl}(W) \rightarrow \mathbb{R}^{n}$

$$
\mathcal{F}(\mathbf{z})=\frac{1}{T} \int_{0}^{T} M_{\mathbf{z}}^{-1}(t, \mathbf{z}, 0) F_{1}(t, \mathbf{x}(t, \mathbf{z}, 0)) d t .
$$

If there exists $a \in V$ with $\mathcal{F}(a)=0$ and $\operatorname{det}((d \mathcal{F} / d \mathbf{z})(a)) \neq 0$, then there exists a $T$ periodic solution $\mathbf{x}(t, \varepsilon)$ of system $(7)$ such that $\mathbf{x}(0, \varepsilon) \rightarrow a$ as $\varepsilon \rightarrow 0$. Moreover, if the eigenvalues of the Jacobian matrix $(d \mathcal{F} / d \mathbf{z})(a)$ are hyperbolic, then the corresponding periodic solution $\mathbf{x}(t, \varepsilon)$ of system ( 7$)$ is hyperbolic and its stability or inestability type is given by those eigenvalues.

Theorem 5 goes back to Malkin [13] and Roseau [14], for a shorter and easier proof see Corollary 1 of [2]. 


\section{Proof of Theorem 1}

We apply the averaging theory described in section 2 for studying the periodic solutions of system (4). More precisely we shall analyze which periodic solutions of system (4) with $\varepsilon=0$ can be continued to periodic solutions of system (4) with $\varepsilon \neq 0$ sufficiently small.

In [9] we studied the solution of system (4) with $\varepsilon=0$. Assuming $a>0$, the general solution of system (4) with $\varepsilon=0$ and with initial conditions $x(0)=x_{0}, y(0)=y_{0}$ is

$$
x(t)=x_{0} \cos \sqrt{a} t-\sqrt{a} y_{0} \sin \sqrt{a} t, \quad y(t)=\frac{\sqrt{a} y_{0} \cos \sqrt{a} t+x_{0} \sin \sqrt{a} t}{\sqrt{a}} .
$$

All solutions of system (4) with $\varepsilon=0$ with the exception of the origin are periodic of period $2 \pi / \sqrt{a}$.

We solve (11) with respect to $\cos \sqrt{a} t$ and $\sin \sqrt{a} t$ and we get

$$
\cos \sqrt{a} t=-\frac{x x_{0}+a y y_{0}}{x_{0}^{2}+a y_{0}^{2}}, \quad \sin \sqrt{a} t=\frac{\sqrt{a} x y_{0}-\sqrt{a} x_{0} y}{x_{0}^{2}+a y_{0}^{2}} .
$$

using the trigonometric relation $\sin ^{2} x+\cos ^{2} x=1$ we get after simplifying the ellipsis

$$
\frac{x^{2}+a y^{2}}{x_{0}^{2}+a y_{0}^{2}}=1 \text {. }
$$

All the solutions of the system (4) with $\varepsilon=0$ with exception of the origin are periodic of period $2 \pi / \sqrt{a}$. These solutions of the unperturbed system fill out the whole plane $\mathbb{R}^{2}$ except the origin. So system (4) with $\varepsilon$ sufficiently small satisfies the assumptions of Theorem 5 .

We write system (4) into the form (7)

$$
\begin{aligned}
& \dot{x}=F_{0,1}(x, y)+\varepsilon F_{1,1}(x, y), \\
& \dot{y}=F_{0,2}(x, y)+\varepsilon F_{1,2}(x, y),
\end{aligned}
$$

where $F_{0}=\left(F_{0,1}, F_{0,2}\right)=(-a y, x)$, and

$$
F_{1}=\left(F_{1,1}, F_{1,2}\right)=\left(y^{3}+h(t, x, y), 0\right) .
$$

The periodic solution $\mathbf{x}(t, \mathbf{z}, 0)$ of system (7) with $\varepsilon=0$ now is the periodic solution $(x(t), y(t))$ given by (11) of system (4) with $\varepsilon=0$ and with initial conditions $\mathbf{z}=$ $\left(x_{0}, y_{0}\right)$. The fundamental matrix $M_{\mathbf{z}}(t)$ of the differential system (9) such that $M_{\mathbf{z}}(0)$ is the identity matrix of $\mathbb{R}^{2}$ is

$$
M_{\mathbf{z}}(t)=\left(\begin{array}{cc}
\cos \sqrt{a} t & -\sqrt{a} \sin \sqrt{a} t \\
\frac{\sin \sqrt{a} t}{\sqrt{a}} & \cos \sqrt{a} t
\end{array}\right) .
$$

By Theorem 5 we must study the zeros $\left(x_{0}, y_{0}\right)$ of the system

$$
\mathcal{F}\left(x_{0}, y_{0}\right)=\left(f_{1}\left(x_{0}, y_{0}\right), f_{2}\left(x_{0}, y_{0}\right)\right),
$$

where according with (10) if we denote $f_{k}=f_{k}\left(x_{0}, y_{0}\right)$ we have

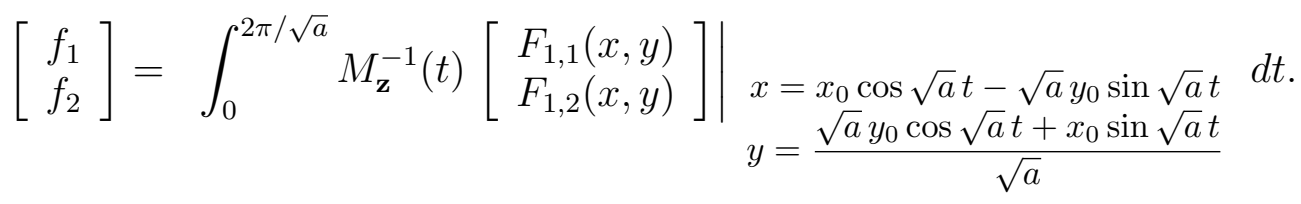


where $F_{1, k}$ for $k=1,2$ are given in (13).

Now, using (13) and (14) in (15), after some simple computations we get the expressions for $f_{1}$ and $f_{2}$ given in (5). Hence, Theorem 5 completes the first part of the proof of Theorem 1 .

The proof of the second part of Theorem 1 on the kind of stability of the periodic solution $(x(t, \varepsilon), y(t, \varepsilon))$ follows directly from the last part of the statement of Theorem 5 .

\section{Proof of the COROLlaries}

In this section we prove the corollaries of Theorem 1.

Proof of Corollary 2. We apply Theorem 1 to system (4) with $h(t, x, y)=\sin \sqrt{a} t$. Then, from (5) we get that

$$
f_{1}\left(x_{0}, y_{0}\right)=\frac{3 y_{0}\left(x_{0}^{2}+a y_{0}^{2}\right)}{8 a^{3 / 2}}, \quad f_{2}\left(x_{0}, y_{0}\right)=-\frac{1}{2 a}-\frac{3 \pi x_{0}\left(x_{0}^{2}+a y_{0}^{2}\right)}{4 a^{5 / 2}} .
$$

We compute the solutions of the system $f_{1}\left(x_{0}, y_{0}\right)=0, f_{2}\left(x_{0}, y_{0}\right)=0$, and we obtain only one real solution:

$$
\left(x_{0}^{*}, y_{0}^{*}\right)=\left(-\frac{2^{2 / 3} \sqrt{a}}{3^{1 / 3}}, 0\right) .
$$

Now, since the determinant (6) takes the value

$$
\frac{3 \cdot 3^{2 / 3}}{8 \cdot 2^{1 / 3} a^{2}} \neq 0
$$

the proof of the corollary follows from Theorem 1.

Proof of Corollary 3. We apply now Theorem 1 to system (4) with $h(t, x, y)=(1+$ $\left.2 x+5 y-5 x^{2}-y^{2}\right) \sin t$. Therefore, from (5) we obtain that

$$
\begin{aligned}
& f_{1}\left(x_{0}, y_{0}\right)=x_{0} y_{0}+\frac{3}{8} y_{0}\left(x_{0}^{2}+y_{0}^{2}\right), \\
& f_{2}\left(x_{0}, y_{0}\right)=-\frac{3}{8} x_{0}\left(x_{0}^{2}+y_{0}^{2}\right)+\frac{1}{2}\left(-1+2 x_{0}^{2}+4 y_{0}^{2}\right) .
\end{aligned}
$$

Then system $f_{1}\left(x_{0}, y_{0}\right)=0, f_{2}\left(x_{0}, y_{0}\right)=0$ has the following five solutions $\left(x_{0}^{*}, y_{0}^{*}\right)$ :

$$
\begin{aligned}
& \left(\frac{8}{9}\left(1-\cos \left(\frac{1}{3} \arctan \left(\frac{9 \sqrt{807}}{13}\right)\right) \pm \sqrt{3} \sin \left(\frac{1}{3} \arctan \left(\frac{9 \sqrt{807}}{13}\right)\right)\right), 0\right), \\
& \left(\frac{8}{9}\left(1+2 \cos \left(\frac{1}{3} \arctan \left(\frac{9 \sqrt{807}}{13}\right)\right)\right), 0\right), \\
& \left( \pm \frac{\sqrt{247}}{32},-\frac{3}{32}\right) .
\end{aligned}
$$

We compute the determinant (6) for the previous five values $\left(x_{0}^{*}, y_{0}^{*}\right)$ and we get that always is different from zero. 


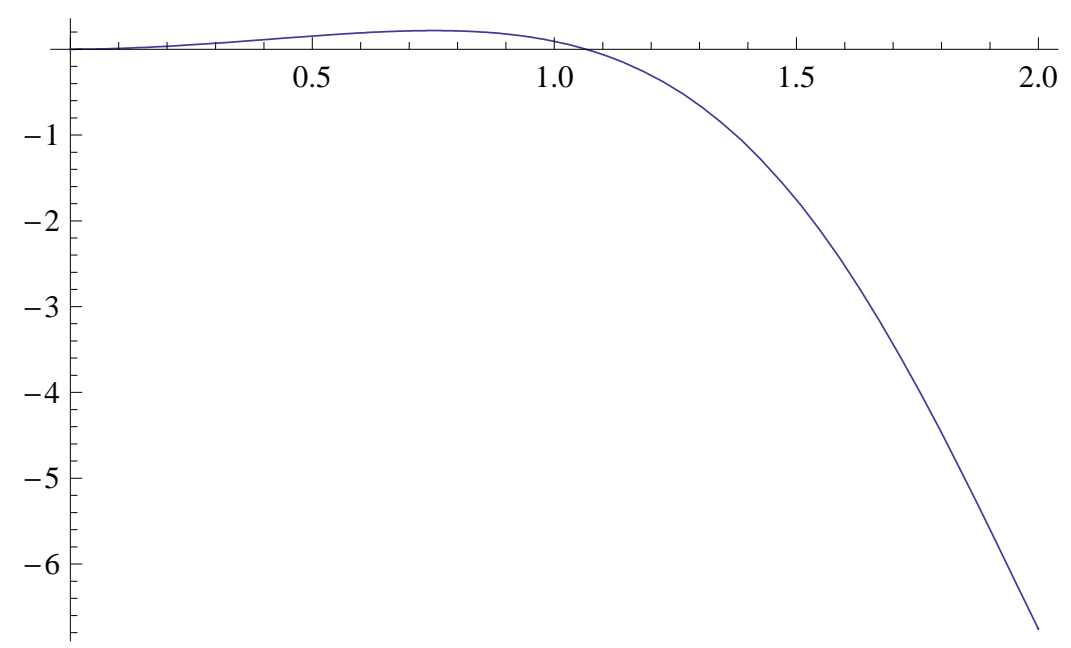

FiguRE 1. Graph of the function (18) as a function of $r$.

Proof of Corollary 4. We apply now Theorem 1 to system (4) with $h(t, x, y)=-\sin \left(x^{2}+\right.$ $\left.y^{2}\right) \sin t$, and we get that

$$
\begin{aligned}
& f_{1}\left(x_{0}, y_{0}\right)=\frac{3}{8} y_{0}\left(x_{0}^{2}+y_{0}^{2}\right), \\
& f_{2}\left(x_{0}, y_{0}\right)=-\frac{3}{8} x_{0}\left(x_{0}^{2}+y_{0}^{2}\right)+\frac{1}{2} \sin \left(x_{0}^{2}+y_{0}^{2}\right) .
\end{aligned}
$$

In order to compute the solutions of the system $f_{1}\left(x_{0}, y_{0}\right)=0, f_{2}\left(x_{0}, y_{0}\right)=0$, we do the change of variables $x_{0}=r \cos \alpha, y_{0}=r \sin \alpha$, and we get that

$$
\begin{aligned}
& f_{1}(r, \alpha)=\frac{3}{8} r^{3} \sin \alpha \\
& f_{2}(r, \alpha)=-\frac{3}{8} r^{3} \cos \alpha+\frac{1}{2} \sin r^{2} .
\end{aligned}
$$

Note that we must have $r \neq 0$, otherwise we would get that $\left(x_{0}, y_{0}\right)=(0,0)$. Thus, $\alpha=0, \pi$.

We substitute $\alpha=0$ in the equation $f_{2}=0$, and we get the equation

$$
-\frac{3}{8} r^{3}+\frac{1}{2} \sin r^{2}=0
$$

Solving it with respect to $r$ we get only one real positive root, providing the solution

$$
\left(r^{*}, \alpha^{*}\right)=(1.0651480242532207 \ldots, 0),
$$

of system (17).

When we substitute $\alpha=\pi$ in the equation $f_{2}(r, \alpha)=0$, we get no real roots.

We finish the proof by computing the determinant (6) which takes the value 13.878369380505733.. $\neq 0$. Hence, the corollary follows from Theorem 1 . 


\section{ACKNOWLEDGEMENTS}

The first author has been supported by the grants MCINN/FEDER MTM200803437, AGAUR 2009SGR 410 and ICREA Academia. The research of AR is supported by the Swedish Research Council (VR Grant 2010/5905). The authors would like to thanks the Göran Gustafsson Foundation UU/KTH for financial support.

\section{REFERENCES}

[1] V.I. Arnold, V.V. Kozlov And A.I. Neishtadt, Mathematical aspects of classical and celestial mechanics, Second Printing, Springer-Verlag, Berlin, 1997.

[2] A. Buică, J.P. Françoise And J. Llibre, Periodic solutions of nonlinear periodic differential systems with a small parameter, Communications on Pure and Applied Analysis 6 (2007), 103111.

[3] H. Chen ANd Y. LI, Stability and exact multiplicity of periodic solutions of Duffing equations with cubic nonlinearities, Proc. Amer. Math. Soc. 135 (2007), 3925-3932

[4] H. Chen AND Y. LI, Bifurcation and stability of periodic solutions of Duffing equations, Nonlinearity 21 (2008), 2485-250

[5] G. Duffing, Erzwungen Schwingungen bei veränderlicher Eigenfrequenz und ihre technisch Bedeutung, Sammlung Vieweg Heft 41/42, Vieweg, Braunschweig, 1918.

[6] J. Guckenheimer and P. Holmes, Nonlinear oscillations, dynamical systems, and bifurcation of vector fields, Springer, 1983.

[7] J.K. Hale AND P.Z. TABOAS, Interaction of damping and forcing in a second order equation, Nonlinear Anal. 2 (1978), 77-84.

[8] G. Hamel, Ueber erzwungene Schingungen bei endlischen Amplituden, Math. Ann. 86 (1922), $1-13$.

[9] J. Llibre AND A. Rodrigues, On the periodic orbits of a kind of Duffing equations, to appear in Appl. Math. Comput.

[10] J. Llibre, M.A. Teixeira And J. Torregrosa, Limit cycles bifurcating from a $k$ dimensional isochronous set center contained in $\mathbb{R}^{n}$ with $k \leq n$, Math. Phys. Anal. Geom. 10 (2007), 237-249.

[11] P. LochaK And C. Meunier, Multiphase averaging for classical systems, Appl. Math. Sciences 72, Springer-Verlag, New York, 1988.

[12] I.G. Malkin, Some problems of the theory of nonlinear oscillations, (Russian) Gosudarstv. Izdat. Tehn.-Teor. Lit., Moscow, 1956.

[13] J. Mawhin, Seventy-five years of global analysis around the forced pendulum equation, Proceedings of Equadiff 9 CD rom, Brno 1997, Masaryk University, pp 115-145.

[14] M. Roseau, Vibrations non linéaires et théorie de la stabilité, (French) Springer Tracts in Natural Philosophy, Vol.8 Springer-Verlag, Berlin-New York, 1966.

[15] J.A. Sanders, F. Verhulst And J. Murdock, Averaging Methods in Nonlinear Dynamical Systems, Second edition, Applied Mathematical Sci. 59, Springer-Verlag, New York, 2007.

[16] F. Verhulst, Nonlinear Differential Equations and Dynamical Systems, Universitext, Springer-Verlag, Berlin, 1996.

[17] X. Wu, J. Li And Y, Zhou, A priori bounds for periodic solutions of a Duffing equation, J. Appl. Math. Comput. 26 (2008), 535-543.

Departament de Matemàtiques, Universitat Autònoma de Barcelona, Bellaterra, 08193 Barcelona, Catalonia, Spain.

E-mail address: jllibre@mat.uab.cat

Matematiska Institutionen, KTH, Lindstedtsvägen 25, S-100 44 Stockholm, SweDEN.

E-mail address: ana.rodrigues@math.kth.se 